(C) 2012 IEEE. Personal use of this material is permitted. Permission from IEEE must be obtained for all other uses, in any current or future media, including reprinting/republishing this material for advertising or promotional purposes, creating new collective works, for resale or redistribution to servers or lists, or reuse of any copyrighted component of this work in other works. 


\title{
A Tongue-Activated Emergency Beacon for Immobile Patients
}

\author{
Sean Sandy and Masood Mehmood Khan
}

\begin{abstract}
This paper reports design and implementation of an infrared signal transmitting tongue activated emergency beacon. This low-cost, simple and reliable device can help immobile patients communicate with the medical staff in the event of an emergency without interfering with other equipment. The physical dimensions of this three sub-system device were minimized to provide flexibility and suit the most vulnerable and impaired patients. The presented sensormicrocontroller configuration results in a robust and intelligent functionality that would allow this device to outperform many of the commercially available systems used in similar environments.
\end{abstract}

\section{INTRODUCTION}

$\mathrm{M}$ ANY hospital patients with a serious illness, disorder or injury enjoy no or limited control over their environment. As a result, they require high amount of care and therefore cause great expense. These patients are unable to perform even simple tasks for their inability to move muscles in a controlled and deliberate manner or due to injuries. They have a reduced range of motion, so something as simple as pressing an emergency help button may not be possible. This requires their carers to be constantly available in case of a voluntary call for help.

Several systems are available for severely ill or injured hospital patients allowing them to communicate or interact with the environment. Some use eye movements as inputs [1] because the majority of spinal cord injury patients are still able to control their eyes. In some systems, eye gaze also gives patients the ability to use a computer or draw using their eyes [2]. There are other systems that use mouth movements to provide inputs to a system, including the sip/puff system that requires the user to blow or suck on a pipe to input [3]. Another such system uses a magnet glued to the patient's tongue, which registers movements via sensors mounted around the patients' face [4].

This paper proposes an Infrared Tongue Activated emergency help Beacon (ITAB) device for receiving input from the demographic similar to the ones targeted by these existing systems. The existing systems are generally complicated and require mounting a large component to the patients' head. This could be undesired and unsatisfactory

Manuscript received October 14, 2011. This work was partially supported by the Department of Mechanical Engineering, Curtin University of Technology, Perth, Western Australia 6012.

Sean Sandy is with A.D. Engineering International Pty Ltd., Malaga WA 6090 (phone: +61 89248 6990; e-mail: seans@ adengineering.com.au).

Masood Mehmood Khan is with Curtin University Department of Mechanical Engineering, Perth, WA 6012 (phone: +61 89266 9205; e-mail: Masood.Khan@curtin.edu.au). for some patients. Due to serious illness or injuries, these patients will already have a large amount of systems, wires, and sensors etc., mounted to their body. Any large or extra equipment on them may be a hindrance in case of an emergency, as it may need to be removed to perform resuscitation or some other procedure.

The proposed ITAB is unobtrusive to both the patient and to the other equipment. Transmission of information between the sensor mounted on the patient and the receiver alarm system takes place via infrared signals. Thus the signals cannot penetrate walls and will not interfere with other equipment. It is also cheap and safe for the patients themselves. The receiver of the signal is flexible enough to allow activation of different emergency systems present in different facilities, including wall mounted 'assistance required buttons', alarms or pager notifications. For these features, the ITAB is more likely to become adopted and carers are expected to prefer it as a long term emergency system.

\section{Tongue Activated DeVICES}

Tongue activated devices are not common yet but some similar systems were developed in the recent past in humancomputer interaction. For example, the tongue drive system (TDS) is an inductive tongue-computer interface that allows disabled people to control the environment with little efforts. It is basically a man machine interface that allows facilitating a number of commands. The system uses 9 sensors. The TDS is designed for relatively immobile patient, who can exercise control on their tongue. Inductive coils are fitted in a palatal plate that fits in the patient's mouth roof and are excited by an activation unit glued to the tongue. Each activated coil is interpreted as a letter in the alphabet, hence the user can type. The induction coils in the patient's mouth are read and transmitted by a lead running out of their mouth, which is then interpreted and displayed. Tests conducted on 3 trained individuals exhibited a possible typing rate in the range $30-57$ characters per minute [4].

TDS is intrusive and uncomfortable for some patients. The palatal plate may; affect breathing, cause some choking hazards, and is expensive to customise. The activation unit glued to the tongue can; cause irritation, rubbing on teeth, catch food particles; and be hard to remove. Leads running out of the patient's mouth may also cause interference with other devices and may hinder staff functionality.

Despite a common demographic, TDS and ITAB have different applications. The ITAB can be used constantly where as the TDS is meant for short-duration uses. 


\section{SYSTEM DESIGN}

The ITAB functions using simultaneous and synchronised operation of two sub-systems. A transmitting sub-system that receives input from a patient and generates an infrared signal. The second component a receiving circuit, receives this signal to activate the hospitals alarm or emergency system. Due to the nature of the intended use, a false positive was preferred over the false negative signal as carers would be closely monitoring a patient using such a device. Following paragraphs explain major components of the system.

\section{A. The Sensing and Transmitting Unit}

A range of sensors could be used with this device. However, a push sensor, requiring a low pressing force, was considered apt for weak patients who experience problems in controlling muscle. A light dependent resistor (LDR) was used for activation when the tongue pressing was observed. As the LDR will have a false positive reading at night, another sensor would activate the system to register pressing and signal the alarms. A thermistor (temperature dependent resistor) was used. It would suit a critical care environment in which the room is likely to be relatively cold and at constant temperature. The patient is usually around $15-20 \mathrm{~K}$ warmer than the room. The thermistor requires constant contact for 1-2 seconds for the resistance to change sufficiently and to ensure that the patient's breathing does not trigger a false positive. A moisture sensor was investigated as it would be a good indication that the patient was deliberately trying to activate the system. For instance it would be able to tell the difference between the patients tongue and cheek.

The employed sensor array included a push button, LRD and thermistor, all in close proximity, so that they all (or at least two) could be pressed easily by a patient. The circuitry will need to take all three inputs and if two of them have positive responses (been pressed, covered or warmed) then an infrared circuit will be activated to communicate with a receiver/ alarm circuit.

The BIR-BL07J4 infrared LED is an end look, light emitting diode [5]. It can project a concentrated beam towards the top. The resultant light beam has a viewing angle of 20 degrees around the axis which is a line projected from the centre of the LED. The Peak wavelength of the emitted light is $940 \mathrm{~nm}$, which is in the near infrared region and perfect for transmitting the SIRC protocol. A range of $12 \mathrm{~m}$ will be ample for a hospital patient, as the patient will rarely, if ever, need more than two meters from the receiver.

A PICAXE 08m microcontroller [6] was chosen for the ITAB. The $08 \mathrm{~m}$ chip has three 8 bit Analogue to Digital Converter (ADC) input/ output pins, one dedicated output $\left(\right.$ infra $\left._{\text {out }}\right)$ and one dedicated input (infra $\left.{ }_{\text {in }}\right)$. The ADC was used so that when the sensors (button, LDR, thermistor) were activated, the program running on the chip would activate the infrared LED.

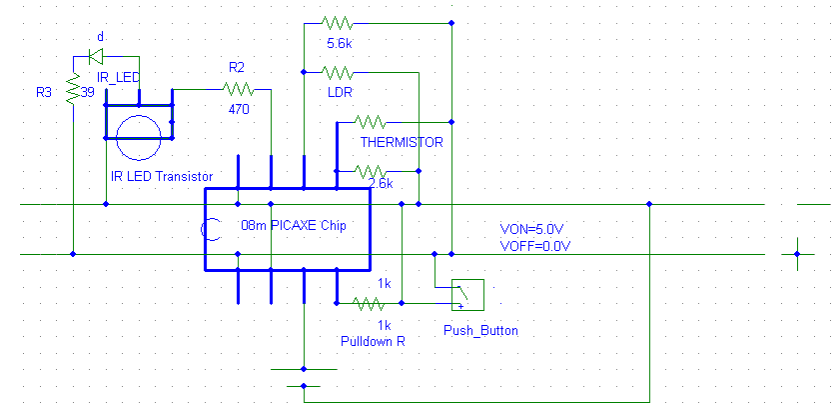

Fig. 1. The transmitter system design.

The infra $\mathrm{out}_{\text {out }}$ andra in $_{\text {ins }}$ and corresponding functions in the program use the SONY SIRC protocol for transmitting via infrared from the sensor-transmitter to the receiver-alarm [7]. This application uses the most basic 12bit SIRC as it transmits a minimum of data. Figure 1 exhibits the transmitter design.

\section{B. Infrared Receiver Unit}

The BRM-15S8-11 [8], a compact infrared receiver was chosen as it could receive remote signals using the SIRC protocol. It had a peak wavelength of 940nm matching that of the infrared LED. Its built-in band pass filter would reject most of the ambient light allowing it to receive the $38 \mathrm{kHz}$ SIRC signal being at the centre frequency. Thus it can filter out any undesired sun, incandescent or extraneous infrared light.

The preamp and Automatic Bias Level Control (ABLC), prevents the input from being saturated by extraneous infrared light, a strong external disturbing light sets the bias level of the input, such that no light data is lost [9]. The limiter takes away the light spectrum outside of the useful infrared region, which the band pass filter (BPF) then filters, to lessen the strength of frequencies outside of the $38 \mathrm{khz}$ SIRC signal. The demodulator extracts the SIRC data from the $38 \mathrm{kHz}$ carrier wave. The integrating op amp amplifies the signal so that the comparator can output a definitive signal comprised of highs (1s) and lows (0s).

The PICAXE microcontroller, programmed in BASIC, has integrated infrared receiving and transmitting capabilities and uses the SIRC protocol.

The configurable $8 / 10$ bit ADCs were used for the sensors. When the DAC reading passes a threshold the sensor is activated. The battery level is also read via a DAC. When the reading passes a threshold, the main battery supply has dropped enough, so that it is nearer to the reference battery. The ADCs allow a large amount of tuning for the sensors so this system can be setup for any environment.

Since the PICAXE output is limited to $20 \mathrm{~mA}$ and the infrared LED requires $50 \mathrm{~mA}$, a transistor is needed. For a current gain of 2.5 at very low power the BD681 transistor was considered apt [9]. Figure 2 shows the receiver system design. 


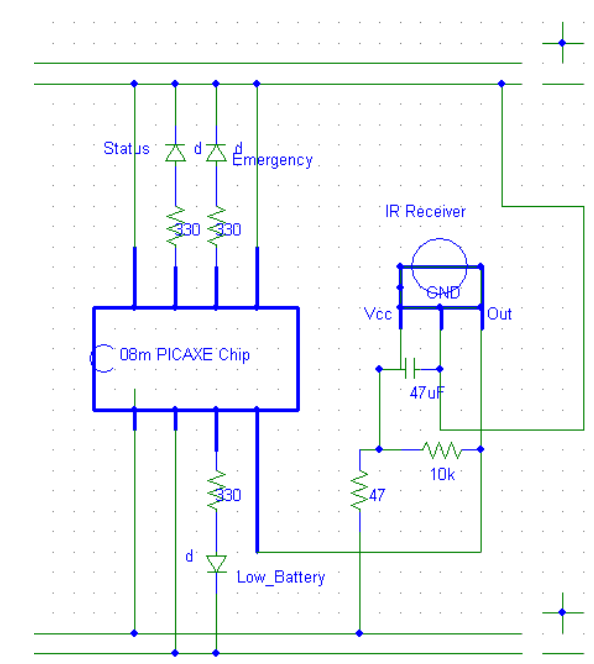

Fig. 2. The receiver system design.

\section{Power Supply}

The system requires $4.5-5$ volt (the working $\mathrm{V}$ range of the PICAXE chip). The supply was meant to be simple, reliable and needed to be on board as any cables running to it could potentially interfere with the other equipment.

A battery with enough capacity could enable device function for a long time between battery changes. Alternately, a solar panel with an open circuit voltage around $1.5 \mathrm{~V}$ higher than the battery could be used for charging the battery. When the voltage of the solar panel is higher than the battery, the diode conducts, charging the battery.

Using coin batteries was the best solution as they provided a good combination of size, weight and cost.

\section{Physical Design And System Mounting}

A major challenge was to design a small, light-weight and flexible system that would easily meet the tongue-activation requirements. After analysing the existing systems, it was decided to mount the device on an oxygen mask, running across the right side of a patients face, roughly across their cheekbone. This positions the device well for transmitting to a receiver placed (where a traditional emergency help button is), over the patient's right shoulder.

The system case was required to be light enough to be mounted on a patient's face, strong enough to support the electronic components inside and to survive a drop onto a hard surface. It needed to be able to withstand saliva, $\mathrm{pH}$ value 6.0-7.4 [10], so it is generally slightly acidic), be easily cleaned with acidic disinfectants commonly used in hospitals and be relatively affordable.

Using an alloy of iron in an acidic environment such as this would rapidly corrode it. Aluminium and PVC are resistant to weak acids so will not corrode in this environment, therefore is likely to last far longer. For these reasons aluminium or medical grade resins were the preferred materials. An aluminium tube, shown in figure 4, was used for the first prototype. This case however was not

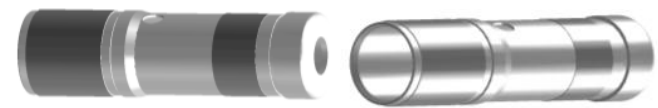

Fig. 4. The prototype case; Top-back, Bottom-Front.

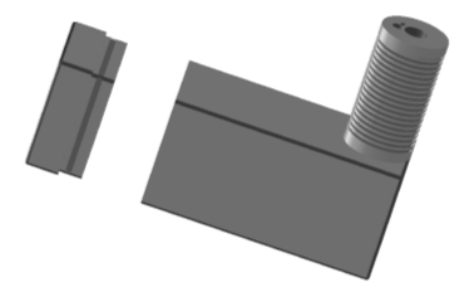

Fig. 5. The adopted case for the ITAB.

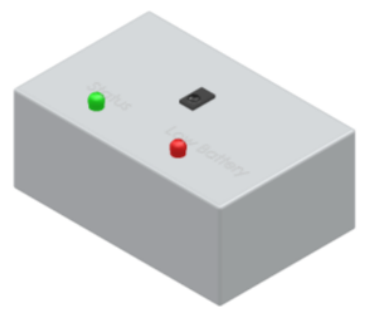

Fig. 6. The ITAB receiver unit.

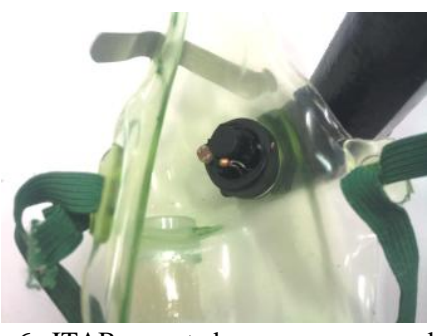

Fig. 6. ITAB mounted on an oxygen mask.

ideal as it would be difficult and expensive to build for the amount of machining required. Shown in Figure 5 is the redesigned and adopted case for the ITAB. The grey box is a machined aluminium case that is made up of a main body and a push to fit lid. The case will hold all the circuitry and batteries, as well as the infrared LED, which protrudes from a hole in the case. Figure 6 shows the receiver unit. Figure 7 shows ITB in a typical operational mode. The operation flowchart and related components of the device are shown in figure 8 .
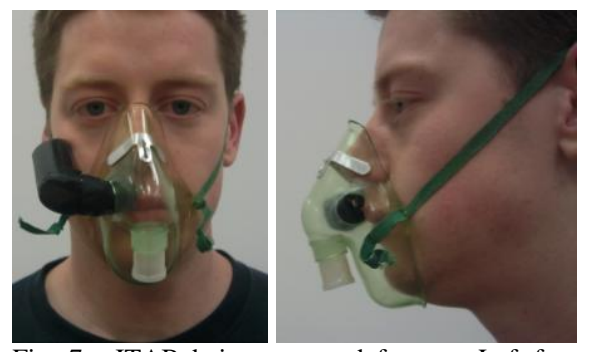

Fig. 7. ITAB being mounted for use. Left-front view; Right-side view. 


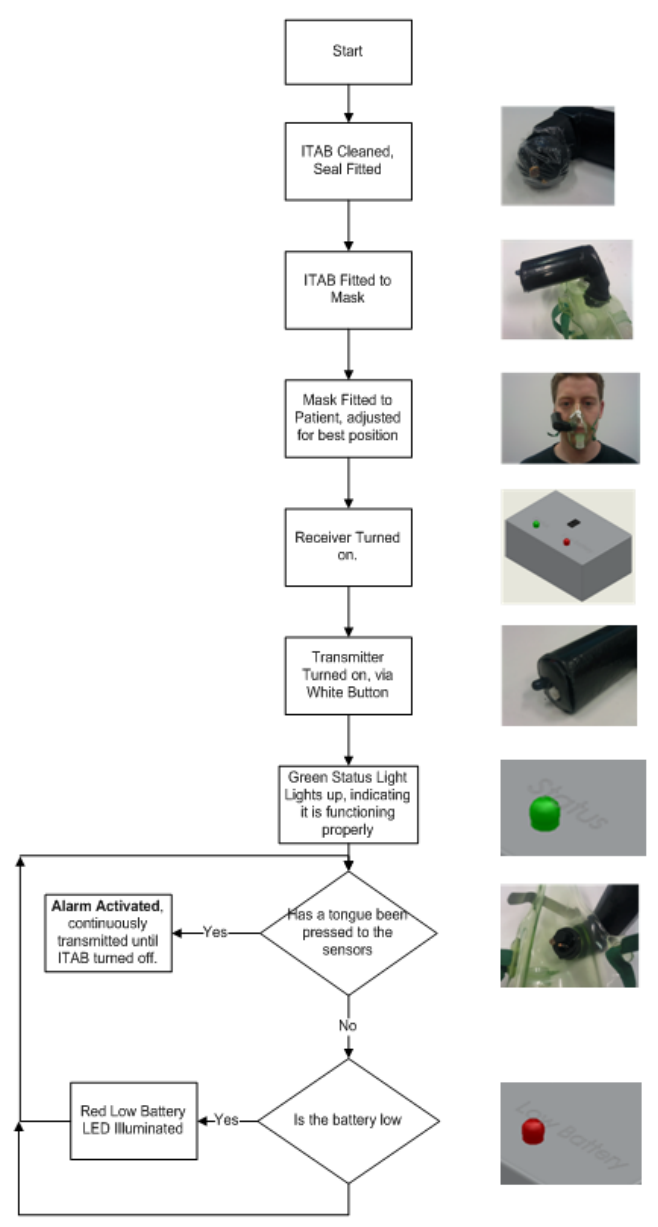

Fig. 8. ITAB's operation flow chart and involved components.

\section{RESULTS}

Initially, the circuit was tested with no user and activated by hand, to test the range. The infrared receiver's range is 12 $\mathrm{m}$ at the ray axis and $6 \mathrm{~m}$ within 45 degrees of it (CORP. 2008). The infrared LED has an effective radiant angle of 20 degrees (CORP.). The test results shown in figure 9 suggest that the system was able to work up to the specified $12 \mathrm{~m}$ range for the receiver. The curve shows that at 2 meters the LED can be facing any direction and will still be received well. In the $3 \mathrm{~m}$ range, the LED needs to be facing at most 90 degrees to the receiver and after that the LED needs to be facing much closer to the receiver to function correctly. The status light function made it very easy to test whether it was in range and aimed correctly. However, it could be deduced that ITAB won't perform under the influence of excessive ambient and extraneous light sources which might corrupt the infrared signal and deteriorate its operational reliably.

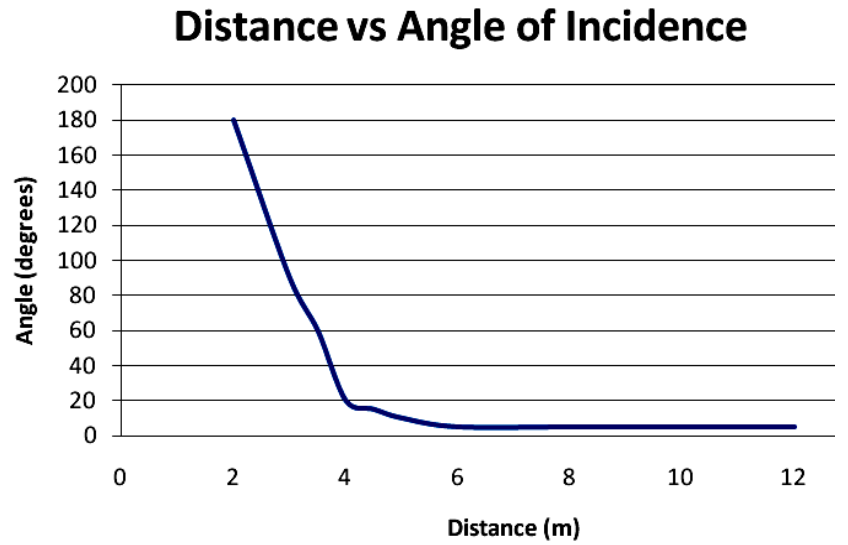

Fig. 9. ITAB mounted on an oxygen mask.

\section{CONCLUSION}

A low cost, convenient and flexible device was developed for impaired patients. It can be activated by a light tongue push, through an infrared signal, for alarm system activation. The final prototype was created after two iterations of circuit operation and an initial prototype, which culminated in optimal design and operation. The circuit used a PICAXE microcontroller which would emit a SIRC infrared protocol via the infrared LED. The device notifies the user when it is working correctly and when the battery needs to be replaced. Being able to quickly reprogram the microcontroller allows for faster and easier development. This is a functionally superior circuit because it is physically much smaller and has far fewer connections. The underlying control program can be changed to tune sensor inputs and operation.

\section{REFERENCES}

[1] The Eyewriter project, CORE development team, 2010, Sept., 27. The EyeWriter. Available : http://www.eyewriter.org/

[2] Eyegaze Edge ${ }^{\mathrm{TM}}$. LC Technologies Inc. 2010, August, 21. Eyegaze Systems. Available : http://www.eyegaze.com/

[3] Sip/Puff Switch. Disabled Online. 2010, August, 11 Available: http://www.disabledonline. com/direct-products/switches/sippuffswitch/

[4] L.N.S.A. Struijk, "An Inductive Tongue Computer Interface for Control Of Computers and assistive Devices" IEEE Trans. Biomed. Eng, vol. 53, no. 12, 2006.

[5] End-Look Package Light Emitting Diode. Bright LED Electronics Corp. 2010, July, 27. BIR-BL07J4 Data Sheet (Infrared LED). Available:

http://www.altronics.com.au/download/Datasheets/Z0880BIRBL07J4.pdf

[6] PICAXE Co. PICAXE Manual. 2010, July, 11. Available: www.picaxe.com/docs/picaxe_manual1.pdf

[7] Sony SIRC infrared protocol. 2010, Feb 10. Available :http://picprojects.org/projects/ sirc/sonysirc.pdf.

[8] Device No. BRM-15S8-11 (Infrared Receiver) Data Sheet. Bright LED Electronics Corp. 2010, July, 11. Available: http://www.altronics.com. au/download/Datasheets/Z1611 A.pdf

[9] IR Preamplifier for Remote Control System. Datasheet4U. 2010, July, 10. Available: http://www.datasheet4u.com/html/D/F/0/ DF0038B Tide.pdf.html.

[10] A.C. Guyton and J.E. Hall. Textbook of Medical Physiology London: Elsevier Health Science Division, 2010. 